\title{
EXTENSION OF COHERENT ANALYTIC SUBSHEAVES
}

YUM-TONG SIU

In this note we prove the following result.

Theorem. Suppose J is a coherent analytic sheaf on a Stein space $(X, \mathfrak{F})$ in the sense of Grauert $[2, \S 1]$ and $S$ is a coherent analytic subsheaf of $\Im \mid U$ for some open neighborhood $U$ of the boundary $\partial X$ of $X$. If for every $x \in U, \varsigma_{x}$, as a $\mathfrak{H}_{x}$-submodule of $\mathfrak{J}_{x}$, has no associated prime ideal of dimension $\leqq 1$, then there exists a coherent analytic subsheaf $\delta^{*}$ of $J$ on $(X, \mathfrak{H})$ such that $\mathrm{S}^{*}$ agrees with $\mathrm{S}$ on some open neighborhood of $\partial X$.

This theorem extends [3, Chapter VII.D.6].

Notations. codh denotes homological codimension. $D(J)$ denotes

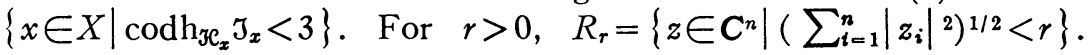
For $s>r>0, R_{r, s}=R_{s}-R_{r}^{-}$, where- denotes topological closure.

Lemma. Suppose $\mathfrak{T} \subset \mathcal{O}(G)^{p}$ is a coherent subsheaf, where $\mathcal{O}(G)$ is the structure sheaf of an open subset $G$ of $\boldsymbol{C}^{n}(n \geqq 3)$ and

$\left(^{*}\right) \mathfrak{M}_{x}$ as an $\mathcal{O}(G)_{x}$-submodule of $\mathcal{O}(G)_{x}^{p}$ has no associated prime ideal of $\operatorname{dim} \leqq 1$ for every $x \in G$.

Then $D(\mathfrak{T})$ is either discrete or empty.

Proof. Suppose not. Since $D(\mathfrak{T C})$ is a subvariety in $G$ [5, Satz 5] there is an irreducible 1-dimensional subvariety $Z$ in a connected Stein open subset $H$ of $G$ such that $Z \subset D(\mathfrak{T})$. Take a holomorphic function $f \not \equiv 0$ on $H$ vanishing on $Z$. Take $x \in Z$. $f_{x} \Re_{x}$ as an $\mathcal{O}(G)_{x}$-submodule of $\mathfrak{M}_{x}$ has no associated prime ideal of dimension $\leqq 1$, for otherwise there is a prime ideal $P$ in $\mathcal{O}(G)_{x}$ of $\operatorname{dim} \leqq 1$ and $s \in \mathfrak{M}_{x}$ such that $s P^{k} \subset f_{x} \mathfrak{M}_{x}$ for some $k$ and $s \notin f_{x} \mathfrak{M}_{x}$. The meromorphic functiongerm $s f_{x}^{-1}$ is holomorphic, because it is holomorphic outside a subvariety-germ of codim $\geqq 2 . s f_{x}^{-1} P^{k} \subset \mathscr{N}_{x}$ and $\left.s f_{x}^{-1} \notin \mathfrak{N}_{x} .{ }^{*}\right)$ is contradicted. Take a holomorphic function $g$ defined in some open neighborhood $W$ of $x$ in $H$ and vanishing on $Z \cap W$ such that $g_{x}$ does not belong to any associated prime ideal of $f_{x} \mathfrak{M}_{x}$ as an $\mathcal{O}(G)_{x}$-submodule of $\mathfrak{M}_{x} . g_{x}$ is not a zero-divisor for $\mathfrak{M}_{x} / f_{x} \mathfrak{M}_{x}$. By coherence of the kernel of the sheaf-homomorphism $\mathfrak{N} / f \mathfrak{T} \rightarrow \mathfrak{N} / f \mathfrak{T}$ defined by multiplication by $g$, after shrinking of $W$ we can assume that $g_{y}$ is not a zero-divisor for $\mathfrak{M}_{y} / f_{y} \mathfrak{T}_{y}$ for $y \in W$. Since $Y=\left\{y \in W \mid \operatorname{codh}(\mathfrak{N} /(f \mathfrak{T}+g \mathfrak{T}))_{y} \leqq 0\right\}$

Received by the editors July 10, 1967 . 
is at most zero-dimensional [5, Satz 5], $\exists z \in(Z \cap W)-Y$. Then $\operatorname{codh} \mathfrak{T}_{z} \geqq 3$. Contradiction, q.e.d.

Proof of Theorem. $K=X-U$ is compact. By replacing $X$ by a relatively compact Stein neighborhood of $K$, we can assume w.l.o.g. that $X$ is a complex subspace of $C^{n}$ with $n \geqq 3$ (Einbettungssatz, [6]) and we have a sheaf-epimorphism $h: \mathcal{O}^{p} \rightarrow \tilde{\jmath}$, where $\tilde{\jmath}$ is the trivial extension of $\boldsymbol{J}$ on $\boldsymbol{C}^{n}$ and $\mathcal{O}$ is the structure sheaf of $\boldsymbol{C}^{n}$. By replacing $X$ by $\boldsymbol{C}^{n}$ and $\delta$ by $h^{-1}(\tilde{\mathcal{S}})$, where $\tilde{\mathcal{S}}$ is the trivial extension of $\mathcal{S}$ on $\boldsymbol{C}^{n}-K$, we can assume w.l.o.g. that $X=C^{n}$ and $J=\mathcal{O}^{p}$. By the lemma we can choose $s>r>d>0$ such that $R_{r-d} \supset K$ and $D(s) \cap R_{r-d, s+d}=\varnothing$. For some $0<a, b<d$, the restriction map $H^{1}\left(R_{r-a, s+b}, \S\right) \rightarrow H^{1}\left(R_{r, s}, \S\right)$ is surjective [1, Propositions 16 and 17, §17]. $\operatorname{dim}_{C} H^{1}\left(R_{r, 8}, \mathcal{S}\right)<\infty$ (cf. the proof of Theorem 11, $[1, \S 17]$ ). Take $x \in R_{r, s}$ and a complexlinear function $f$ on $\boldsymbol{C}^{n}$ such that $f(x)=0$ and the set $V$ of zeroes of $f$ is disjoint from $R_{r}^{-}$. The exact sequence $0 \rightarrow S \stackrel{u}{\rightarrow} S \rightarrow S / f S \rightarrow 0$, where $u$ is defined by multiplication by $f$, yields the exact sequence $\Gamma\left(R_{r, s}, s\right)$ $\stackrel{n}{\rightarrow} \Gamma\left(R_{r, s}, \quad \delta / f \S\right) \rightarrow H^{1}\left(R_{r, s}, \quad \delta\right) \rightarrow H^{1}\left(R_{r, s}, \quad \delta\right) \rightarrow H^{1}\left(R_{r, s}, \quad \delta / f \delta\right)$. Since $V \cap R_{r, s}$ is Stein, $H^{1}\left(R_{r, s}, \delta / f S\right)=0 . \operatorname{dim}_{C} H^{1}\left(R_{r, s}, \S\right)<\infty$ implies that $v$ is surjective. Let $m$ be the maximal ideal of $\mathcal{O}_{x} . w: \Gamma\left(R_{r, s}, \delta / f \delta\right)$ $\rightarrow \mathrm{S}_{x} / m \mathrm{~S}_{x}$ is surjective, because $V \cap R_{r, s}$ is Stein. $w \circ v$ is surjective. By Krull-Azumaya Lemma [4, (4.1)], $\Gamma\left(R_{r, s}, \delta\right)$ generates $\delta_{x}$. Since $x$ is arbitrary, $\delta$ restricted to $R_{r, 8}$ is generated by sections on $R_{r, 8}$. Extensions of elements of $\Gamma\left(R_{r, s}, \S\right)$ form a subset $S$ of $\Gamma\left(R_{s}, \mathcal{O}^{p}\right)$. $S$ generates a coherent subsheaf $\mathcal{F}$ of $\mathfrak{J}$ on $R_{s}$. Define $\mathcal{S}^{*}$ to be $\mathcal{F}$ on $R_{s}$ and to be $S$ on $C^{n}-R_{r}^{-}$. Then $\mathcal{S}^{*}$ is the required extension, q.e.d.

\section{REFERENCES}

1. A. Andreotti and $\mathrm{H}$. Grauert, Théorèmes de finitude pour la cohomologie des espaces complexes, Bull. Soc. Math. France. 90 (1962), 193-259.

2. H. Grauert, Ein Theorem der analytischen Garbentheorie und die Modulräume komplexer Strukturen, Inst. Hautes Études Sci. Publ. Math. 5 (1960).

3. R. C. Gunning, and H. Rossi, Analytic functions of several complex variables, Prentice-Hall, Englewood Cliffs, N. J., 1965.

4. M. Nagata, Local rings, Interscience, New York, 1962.

5. G. Scheja, Fortsetzungssätze der komplex-analytischen Cohomologie und ihre algebraische Charakterisierung, Math. Ann. 157 (1964), 75-94.

6. K.-W. Wiegmann, Einbettungen komplexer Räume in Zahlenräume, Invent. Math. 1 (1966), 229-243.

University of Notre DAme 